\title{
The measurement of the pp chain solar neutrinos in Borexino
}

\author{
Gemma Testera* \\ Istituto Nazionale di Fisica Nucleare (Genova) Italy \\ E-mail: testera@ge.infn.it \\ On behalf of the Borexino Collaboration: \\ M. Agostini ${ }^{m}$, K. Altenmüller ${ }^{m}$, S. Appe ${ }^{m}$, V. Atroshchenko ${ }^{g}, Z_{\text {Z. Bagdasarian }}{ }^{j u}$, D. Basilico $^{h}$, G. Bellini ${ }^{h}$, J. Benziger $^{k c}$, \\ G. Bonfini ${ }^{e}$, D. Bravo ${ }^{h}$, B. Caccianiga ${ }^{h}$, F. Calaprice ${ }^{k}$, A. Caminata ${ }^{c}$, A. Cappelli ${ }^{e}$, S. Caprioli ${ }^{h}$, M. Carlini ${ }^{e}$,

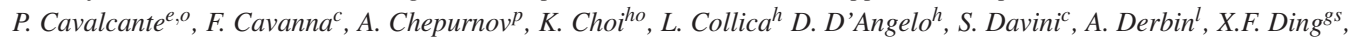

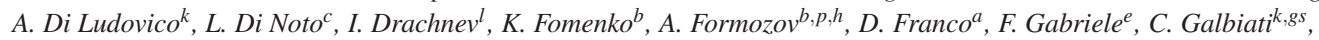

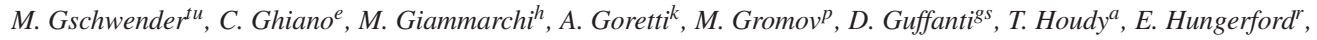 \\ Aldo Ianni ${ }^{e}$, Andrea Ianni ${ }^{k}$, A. Jany ${ }^{d}$, D. Jeschke ${ }^{m}$, V. Kobyche ${ }^{k i}$, G. Korga ${ }^{r}$, S. Kumaran ${ }^{j u, r w}$, T. Lachenmaier ${ }^{\text {tu }}$, \\ M. Laubenstein ${ }^{e}$, E. Litvinovich ${ }^{f, g}$, P. Lombardi ${ }^{h}$, L. Ludhova ${ }^{j u, r w}$, G. Lukyanchenko ${ }^{f}$, L. Lukyanchenko $^{f}$, \\ I. Machulin ${ }^{f, g}$, G. Manuzioc, S. Marcocci ${ }^{s s}$, J. Maricicho ${ }^{\text {ho }}$ J. Martyn ${ }^{m z}$, E. Meroni ${ }^{h}$, M. Meyer ${ }^{d d}$, L. Miramonti ${ }^{h}$, \\ M. Misiaszek ${ }^{d}$, V. Muratoval ${ }^{l}$, B. Neumair ${ }^{m}$, M. Nieslony ${ }^{m z}$, L. Oberauer $^{m}$, V. Orekhov ${ }^{f}$, F. Ortica ${ }^{j}$, M. Pallavicini $^{c}$, \\ L. Papp ${ }^{m}$, Ö. Penek ${ }^{j u, r w}$, L. Pietrofaccia ${ }^{k}$, N. Pilipenko ${ }^{l}$, A. Pocar ${ }^{n}$, A. Porcelli ${ }^{m z}$, A. Raikov $^{f}$, G. Ranucci ${ }^{h}$, A. Razeto ${ }^{e}$

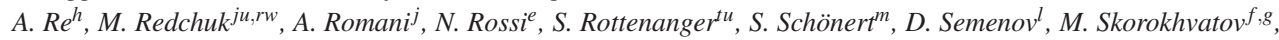 \\ O. Smirnov ${ }^{b}$, A. Sotnikov ${ }^{b}$, L.F.F. Stokes ${ }^{e}$, Y. Suvorov ${ }^{e, f}$, R. Tartaglia ${ }^{e}, J$. Thurn $^{\text {dd }}$, E. Unzhakov ${ }^{l}$, F. Villante Seq $^{e}$,

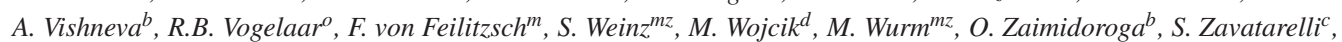 \\ K. Zuber dd, G. Zuzel ${ }^{d}$
}

[a] APC, Univ. Paris Diderot, CNRS/IN2P3, CEA/Irfu, Obs. de Paris Sorbonne Paris Cité, France

[b] Joint Institute for Nuclear Research, Dubna 141980, Russia

[c] Dipartimento di Fisica, Università e INFN, Genova 16146, Italy

[he] Max-Planck-Institut für Kernphysik, 69117 Heidelberg, Germany

[d] M. Smoluchowski Institute of Physics, Jagellonian University, Krakow, 30059, Poland

[e] INFN Laboratori Nazionali del Gran Sasso, Assergi 67010, Italy

[f] NRC Kurchatov Institute, Moscow 123182, Russia

[g] National Research Nuclear University MEPhI (Moscow Engineering Physics Institute), 115409 Moscow, Russia [h] Dipartimento di Fisica, Università degli Studi e INFN, Milano 20133, Italy

[i] Max-Plank-Institut für Kernphysik, Heidelberg 69029, Germany

[j] Dipartimento di Chimica, Università e INFN, Perugia 06123, Italy

[k] Physics Department, Princeton University, Princeton, NJ 08544, USA

[kc] Chemical Engineering Department, Princeton University, Princeton, NJ 08544, USA

[ki] Kiev Institute for Nuclear Research, 03680 Kiev, Ukraine

[l] St. Petersburg Nuclear Physics Institute, Gatchina 188350, Russia

[m] Physik Department, Technische Universität München, Garching 85747, Germany

[n] Physics Department, University of Massachusetts, Amherst MA 01003, USA

[o] Physics Department, Virginia Polytechnic Institue and State University, Blacksburg, VA 24061, USA

[p] Lomonosov Moscow State University, Institute of Nuclear Physics, Moscow 119234, Russia

[q] Institut für Experimentalphysik, Universität Hamburg, Germany

[r] Department of Physics, University of Houston, Houston, TX 77204, USA

[s] Physics ans Astronomy Department, University of California Los Angeles (UCLA), Los Angeles, CA 90095, USA [t] Eberhard Karls Universität Tübingen, Physikalisches Institut, auf der Morgenstelle 14, 72076 Tb̈ingen, Germany [gs] Gran Sasso Science Institute (INFN), 67100 L'Aquila, Italy

[ju] Institut für Kernphysik, Forschungszentrum Jülich, 52425 Jülich, Germany

[rw] RWTH Aachen University, 52062 Aachen, Germany

[ho] Department of Physics and Astronomy, University of Hawaii, Honolulu, HI 96822, USA

[tu] Kepler Center for Astro and Particle Physics, Universität Tübingen, 72076 Tübingen, Germany

[mz] Inst. of Phys. and Excellence Cluster PRISMA, Johannes Gutenberg-Universität Mainz, 55099 Mainz, Germany [dd] Department of Physics, Technische Universität Dresden, 01062 Dresden, Germany

[aq] Dipartimento di Scienze Fisiche e Chimiche, Università dell'Aquila, 67100 L'Aquila, Italy 
Borexino recently performed a comprehensive measurement of the solar neutrino fluxes of whole $p p$ chain. We outline the interest and some physical consequence of the results.

Neutrino Oscillation Workshop (NOW2018)

9 - 16 September, 2018

Rosa Marina (Ostuni, Brindisi, Italy)

\footnotetext{
* Speaker.

${ }^{\dagger}$ Present address : Universidad Autónoma de Madrid, Ciudad Univer. de Cantoblanco, 28049 Madrid, Spain

$\dagger \dagger$ Present address: INFN LNGS, 67010 Assergi (AQ), Italy

$\dagger \dagger$ Present address: INFN LNGS, 67010 Assergi (AQ), Italy

$\dagger+$ Also at: MTA-Wigner Res. Centre for Phys., Dep. Space Phys. and Tech., 1121 Budapest, Hungary

$\dagger-$ Present address: Fermilab National Accelerato Laboratory (FNAL), Batavia, IL 60510, USA

$\dagger^{+-}$Present address: Dipartimento di Fisica, Sapienza Università di Roma e INFN, 00185 Roma, Italy

$\dagger^{++}$Present address: Dipartimento di Fisica, Università degli Studi Federico II e INFN, 80126 Napoli, Italy
} 


\section{Introduction}

About $99 \%$ of the energy of the Sun is produced through nuclear fusion $[1,2]$ in which hydrogen is converted into helium through a sequence of nuclear reactions (named pp-chain) initiated by the proton proton $(p p)$ or by the proton electron proton reaction ( $p e p)$. The fusion develops through 3 branches (named ppI-ppII-ppIII) and electron neutrinos $v$ are produced in five different type of reactions and they are labelled as pp, pep, ${ }^{7} \mathrm{Be},{ }^{8} \mathrm{~B}$ and hep solar neutrinos. ${ }^{7} \mathrm{Be} v$ 's $\left(\mathrm{E}_{v}=0.384 \mathrm{MeV}\right.$ and $0.862 \mathrm{MeV})$ and pep $v$ 's $\left(\mathrm{E}_{v}=1.44 \mathrm{MeV}\right)$ are mono-energetic line, while the others components show a continuous spectrum dominated by the most abundant $p p v\left(\mathrm{E}_{v}<0.42 \mathrm{MeV}\right)$ and extending up to $\sim 18.8 \mathrm{MeV}$ (maximum energy of the hep $v$ ). In addition the remaining contribution to the Sun energy is produced by another sequence of nuclear reactions in which $\mathrm{C}, \mathrm{N}, \mathrm{O}$ act as catalysts (the $\mathrm{CNO}$ cycle) and that originates neutrinos with a continuous spectrum extending up to $1.74 \mathrm{MeV}$. The CNO contribution to the energy production in the Sun is only of the order of $1 \%$ but, on the contrary, it represents an important component for the dynamics of stars heavier than the Sun.

After many years since their first detection, solar neutrinos are still a source of important information both in astrophysics and in neutrino physics. The standard solar model predicts the spectrum of solar neutrinos but the value of the flux of the various components is still a subject of scientific debate inside the astrophysics community. The so called High Metallicity (HZ) [3] and Low Metallicity (LZ) [4] models predict different fluxes, in particular for the neutrinos of the sub-dominant $\mathrm{CNO}$ cycle but also for the ${ }^{7} \mathrm{Be}$ and ${ }^{8} \mathrm{~B}$ components [5]. The precise measurement of the neutrinos fluxes can help to discriminate between these models. Furthermore solar neutrinos also offer a way to deeply probe the models of neutrino oscillation and eventually to single out or to constrain hypothesis about not standard neutrino interactions [9]. The Sun produces electron neutrinos but they reach the Earth as a mixture of all flavours. Matter oscillations in the Sun play a key role (MSW effect) [6,7] in the oscillation process. The resulting electron neutrino survival probability $P_{e e}$, according to the LMA-MSW solution [8] of the oscillation scenario, depends on the neutrino energy. Non standard neutrino interactions [9] significantly modify the shape of this energy dependence. The precise measurement of all the solar neutrinos fluxes is a powerful tool to confirm or not the prediction of the LMA-MSW scenario.

Using the data of the Phase II (that is the ones following a purification in situ of the scintillator, see below) of Borexino the complete study of all the neutrino components of the pp-chain has been performed [10]: we measured the interaction rates of $p p,{ }^{7} \mathrm{Be}$, and pep neutrino with the highest precision to date [11], and of ${ }^{8} \mathrm{~B}$ neutrinos with the lowest-threshold [12]. We also set a limit on the neutrino flux originated by the reaction of ${ }^{3} \mathrm{He}$ and proton named hep. These measurements provide a direct determination of the relative intensity of the two primary terminations of the ppchain (pp-II/pp-I ) and a first indication that the temperature profile in the Sun is more compatible with solar models assuming high surface metallicity. At the same time, we determined the survival probability $P_{e e}$ of solar electron neutrinos at different energies, thus probing simultaneously and with high precision the solar neutrino flavor conversion mechanism. 


\section{The detector and the data analysis}

Borexino is collecting data since May 2007 in the Laboratory Nazionali del Gran Sasso (Italy). The principle of the detector design is very simple: it basically consists of a of $\sim 278$ ton of organic liquid scintillator contained within a sphere of $4.25 \mathrm{~m}$ diameter, viewed by 2200 photomultipliers and shielded against the external radioactivity. Details of the detector are described in [13]. What makes this instrument extremely powerful for performing low energy solar neutrino spectroscopy are the unprecedented low levels of background that have been achieved after several years of research and efforts. The initial radio-purity of the detector exceeded the design goal for several contaminants, however after May 2010 we began a series of procedures to purify the scintillator aiming to further reduce the background with the goal of increasing the sensitivity of the solar neutrino detection. We call solar Phase-II the data taking period following the end of this purification campaign. This purification campaign includes 6 cycles of closed-loop water extraction and it has significantly reduced the radioactive contaminants: ${ }^{238} \mathrm{U}<9.4 \times 10^{-20} \mathrm{~g} / \mathrm{g}\left(95 \%\right.$ C.L.), ${ }^{232} \mathrm{Th}$ $<5.7 \times 10^{-19} \mathrm{~g} / \mathrm{g}(95 \%$ C.L. $),{ }^{85} \mathrm{Kr}$ and ${ }^{210} \mathrm{Bi}$ have been reduced respectively by a factor $\sim 4.6$ and $\sim 2.3$ compared to the values reached in the first phase (Phase I) of the data taking.

Neutrinos interact through elastic scattering with electrons in the liquid scintillator target. There is no particular signature that allows to distinguish between neutrino induced events and background due to the radioactive decay of isotopes contaminating the scintillator and the surrounding materials. The expected solar neutrino event rate of few tens or few events per day in 100 tons sets the requirements about the needed radio-purity. The true signal is extracted from the background by fitting global observables (the energy spectrum, the radial distribution of the events and suitable distribution of pulse shape variables) of the detected events.

The physical observables reconstructed from the PMTs signals are the electron energy (related to the amount of detected light, about $\sim 500$ photoelectrons $/ \mathrm{MeV}$ ) and the position of the interaction inside the scintillator (related to the difference of the arrival time of the signals on different PMTs). As a reference at $1 \mathrm{MeV}$, the energy and position reconstruction resolution $\sigma$ are $\sim 50 \mathrm{Kev}$ and $\sim 10 \mathrm{~cm}$ respectively.

An event occurs when a a group of PMTs fires within $100 \mathrm{~ns}$, corresponding to an hardware energy threshold of about $50 \mathrm{KeV}$. We define 3 energy regions for the analysis: the first one (called Low Energy Region, LER) contains events with the lowest energy, from $0.19 \mathrm{MeV}$ to $2.93 \mathrm{MeV}$ and it is used to measure the interaction rate of $p p,{ }^{7} \mathrm{Be}$ and pep $v$, the second one is called High Energy Region 1 (HER-I) and includes events with energy from 3.2 to $5.7 \mathrm{MeV}$ and finally the last one, called High Energy Region 2 (HER-II), spans the energy interval from 5.7 to $17 \mathrm{MeV}$. The regions HER-I and HER-II are used to measure the ${ }^{8} \mathrm{~B}$ interaction rate. Note that the lower edge of the HER-I region defines the energy threshold for the measurement of the ${ }^{8} \mathrm{~B} v$ flux: it cannot be reduced below 3.2 MeV because of the background of $2.614 \mathrm{MeV} \gamma$ of the ${ }^{208} \mathrm{Tl}$ decay due to the contamination of ${ }^{232} \mathrm{Th}$ of the PMTs and the support sphere.

Before performing the fit procedure there are a few powerful handles allowing to discriminate the signal from the background. One of this is the fast time response of the scintillator that allows to reconstruct the position of the events and thus to define a wall less fiducial volume where the background events are sufficiently suppressed. We have defined different fiducial volumes (FV) depending on the particular signal that we wanted to extract: in the LER we selected a 71.3 tons 
FV through a radial and $z$ cut needed to suppress $\gamma$ background from contaminants of the external part of the detectors, while a larger FV is used for the ${ }^{8} \mathrm{~B}$ search. A second important handle is the pulse shape discrimination due to the different time response of the scintillator to the energy deposit of different type of particles. This feature allows to suppress events due to $\alpha$ decay and also to discriminate between energy deposit due to electrons (or $\gamma$ ) and positrons. The analysis proceeds in two steps: i) the event selection, with a optimised set of cuts in the three energy regions to maximize the signal-to-background ratio, and ii) the extraction of the neutrino and residual background rates with a combined fit of distributions of global quantities built for the events surviving the cuts. We used a multivariate approach in the LER fitting simultaneously the energy spectrum, the radial distribution of the events and a pulse shape parameter helpful to suppress ${ }^{11} \mathrm{C}$ background events while we fit only the radial distributions for the extraction of ${ }^{8} \mathrm{~B}$.

The unique Borexino measurement of all the solar $v$ fluxes of the pp chain spanning from the low energy of $0.19 \mathrm{MeV}$ (dominated by pp $v$ ) up to the end point of ${ }^{8} \mathrm{~B} v$ of $17 \mathrm{MeV}$ demands a deep understanding of backgrounds, and an accurate modeling of the detector response. These have been achieved through a precise ab initio simulation with a Monte Carlo method [14] and with analytical models [15]. In situ calibration of the detector with radioactive sources has allowed to validate the accuracy of the Monte Carlo. The Monte Carlo model reproduces the geometry of the detector, the energy loss of every particle in every material and it generates and propagates the scintillation and Cerenkov light until it is detected by the PMTs, including all the optical processes in the scintillator and the response of the electronic chain processing the PMT signal. Time variation of the detector response due the a variable number of active PMTs and deviations of the shape of the nylon vessel from the ideal spherical shape are included in the simulation.

\section{Main results}

We refer to [11,12] for details of the analysis and to [10] for a full discussion of the results. Here we only recall and show the two most relevant consequences of the measurements. The first one is the determination of the solar electron neutrino survival probability $P_{e e}$ over the entire energy spectrum that is is shown in figure 1.

The second one is the hint about the Sun metallicity obtained with the Borexino data. We use only the results for ${ }^{7} \mathrm{Be}$ and ${ }^{8} \mathrm{~B}$ neutrinos, whose fluxes display a significant difference between HZ and LZ- SSM theoretical predictions (9\% and 18\%, respectively). Figure 2 shows the results of Borexino (green shaded ellipse), together with the predictions for the HZ and LZ standard solar models (blue and red shaded ellipses, respectively). Note that the errors in the Borexino measurements are in both cases smaller than the theoretical uncertainties. The theoretical error budget is dominated by uncertainties on the astrophysical factor of nuclear reactions and on the opacity of the Sun.

While the Borexino results are compatible with the temperature profiles predicted by both $\mathrm{HZ}$ and LZ, they provide an interesting hint in favor of the HZ prediction. A frequentist hypothesis test based on a likelihood-ratio test statistics (HZ vs LZ) was performed by computing the probability distribution functions with a toy Monte Carlo approach. Assuming HZ to be true, our data disfavor LZ at $96.6 \%$ C.L. This constraint is slightly stronger than our sensitivity (the median sensitivity is at $94.2 \%$ C.L.). We have also performed a global fit including the results presented in this work 


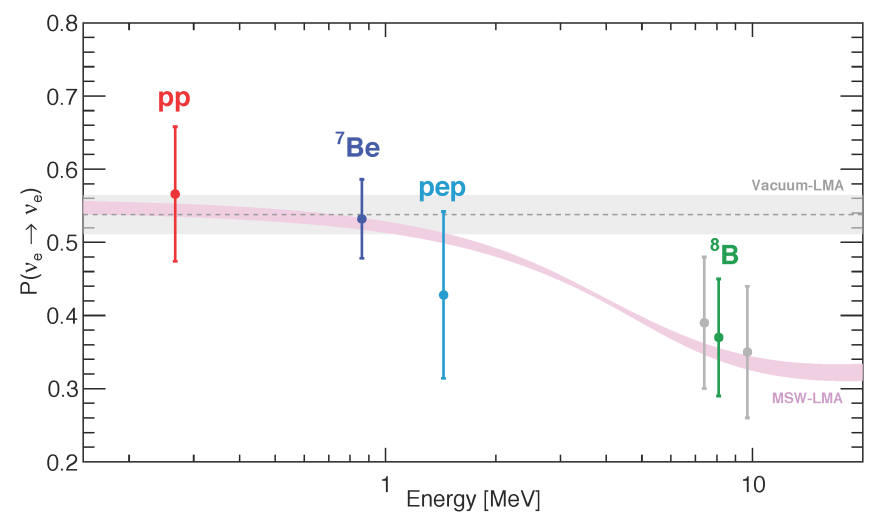

Figure 1: $P_{e e}$ for $v$ 's from the $p p$ chain in the Sun as measured by Borexino. The violet band corresponds to the $\pm 1 \sigma$ prediction of the MSW-LMA solution [8]. The vertical error bars of each data point represent the $\pm 1 \sigma$ interval (experimental + theoretical); the horizontal bar shows the neutrino energy range spanned by the $v$ component under consideration. For ${ }^{7} \mathrm{Be}$ neutrinos the error is dominated by the theoretical uncertainty, while for $p p$, pep, and ${ }^{8} \mathrm{~B}$ the error is dominated by the experimental one.

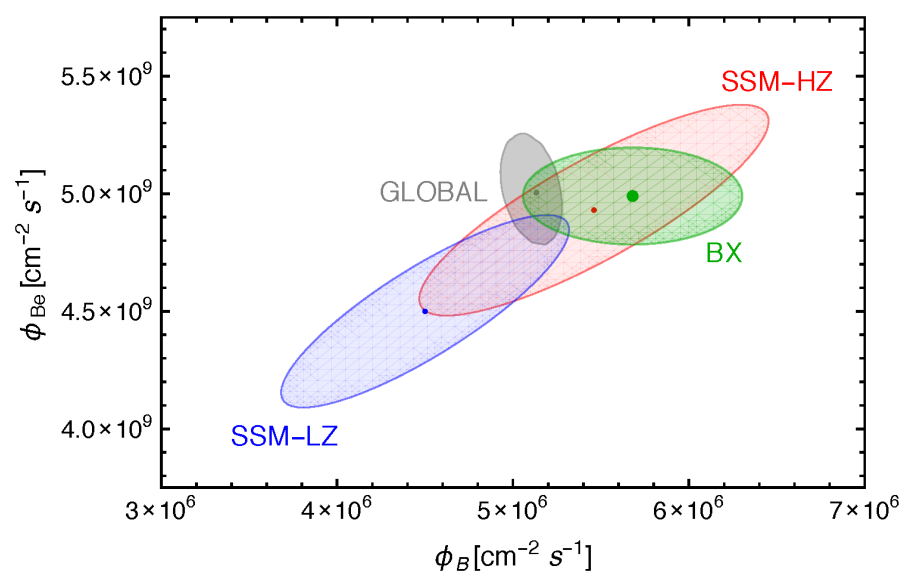

Figure 2: Results about the metallicity studies with our ${ }^{7} \mathrm{Be}$ and ${ }^{8} \mathrm{~B}$ measured neutrino fluxes (green point and shaded area around it). We show in addition the allowed contours obtained by combining these new results with all solar and KamLAND data, and leaving free the oscillation parameters. The theoretical prediction for the low metallicity (LZ) (blue) and the high metallicity (HZ) (red) Standard Solar Models are also shown. All contours correspond to $68.27 \%$ C.L.

together with all the other solar plus KamLAND data: when all the results are combined together the hints toward the HZ further weakens.

\section{Conclusions}

After more than 10 years of data taking and efforts both in the purification of the scintillator and in the data analysis, calibration and detector modeling, Borexino provided a measurement of 
the entire spectrum of all the solar component of the pp chain with a unified analysis. These results extend the previous ones already obtained during Phase I [16-22] and improve their accuracy.

\section{References}

[1] W.A. Fowler, Astrophys. J. 127 (1958) 551.

[2] A.W.G. Cameron, Ann. Rev. Nucl. Sci. 8 (1958) 299.

[3] A.M. Serenelli, W.C. Haxton, and C. Peña-Garay, Astrophys. J. 743 (2011) 24.

[4] M. Asplund et al., Astrophys. J. Lett. 705 (2009) L123.

[5] N. Vinyoles et al., Astrophys. J. 835 (2017) 202.

[6] L. Wolfenstein, Phys. Rev. D 172369 (1978).

[7] S. P. Mikheyev, A. Yu. Smirnov, Sov. J. Nucl. Phys. 42913 (1985).

[8] I. Estebanet al., JHEP 1701 (2017) 087.

[9] A. Friedland et al., Phys. Lett. B 594 (2004) 347; S. Davidson et al., JHEP 03 (2003) 011; P.C. de Holanda and A.Yu. Smirnov, Phys. Rev. D 69 (2004) 113002; A. Palazzo and J.W.F. Valle, Phys. Rev. D 80 (2009) 091301.

[10] M. Agostini et al. (Borexino Collaboration), Nature 562 (2018) 505.

[11] M. Agostini et al. (Borexino Collaboration), arXiv:1707.09279.

[12] M. Agostini et al. (Borexino Collaboration), arXiv:1704.02291.

[13] G. Alimonti et al. (Borexino Collaboration), Nucl. Instrum. Methods Phys. Res. A 600 (2009) 568.

[14] M. Agostini et al., (Borexino Collaboration), Astropart. Phys. 97 (2018) 136.

[15] G. Bellini et al., Phys. Rev. D 89 (2014) 112007.

[16] C. Arpesella. et al. (Borexino Collaboration), Phys. Lett. B 658 (2008) 101.

[17] C. Arpesella et al. (Borexino Collaboration), Phys. Rev. Lett. 101 (2008) 091302.

[18] G. Bellini et al. (Borexino Collaboration), Phys. Rev. Lett. 107 (2011) 141302.

[19] G. Bellini et al. (Borexino Collaboration), Phys. Lett. B 707 (2012) 22.

[20] G. Bellini et al. (Borexino Collaboration), Phys. Rev. Lett. 108 (2012) 051302.

[21] G. Bellini et al. (Borexino Collaboration), Phys. Rev. D 82033006 (2010).

[22] G. Bellini et al. (Borexino Collaboration), Nature 512 (2014) 383. 